\title{
De Luther à la loi Debré : protestantisme, école et laïcité
}

From Luther to the "loi Debré": Protestantism, Education and Secularism

Von Luthers Theorie zum Debré-Gestetz: Protestantismus, Schulwesen und

Laizität

De Lutero a la ley Debré: protestantismo, escuela y laicidad

\section{Patrick Cabanel et André Encrevé}

\section{OpenEdition}

Journals

Édition électronique

URL : https://journals.openedition.org/histoire-education/1341

DOI : $10.4000 /$ histoire-education. 1341

ISSN : 2102-5452

Éditeur

ENS Éditions

Édition imprimée

Date de publication : 1 mai 2006

Pagination : $5-21$

ISBN : 2-7342-1066-5

ISSN : 0221-6280

Référence électronique

Patrick Cabanel et André Encrevé, « De Luther à la loi Debré : protestantisme, école et laïcité », Histoire de l'éducation [En ligne], 110 | 2006, mis en ligne le 01 janvier 2011, consulté le 20 mai 2021. URL : http://journals.openedition.org/histoire-education/1341; DOI : https://doi.org/10.4000/histoireeducation.1341 


\title{
De Luther à la loi Debré: PROTESTANTISME, ÉCOLE ET LAICITÉ
}

\author{
par Patrick CABANEL et André ENCREVÉ
}

Il est clair que le protestantisme entretient un rapport particulier avec l'éducation. En effet, en insistant sur l'obligation de connaître la doctrine chrétienne non plus par transmission orale mais par l'intermédiaire de la lecture de la Bible, Luther, comme l'écrivent François Furet et Jacques Ozouf, «rend nécessaire ce que Gutenberg a rendu possible» (1). En insistant sur le principe dit du sola scriptura, dans le but de s'opposer à toute une série de doctrines et de pratiques catholiques qu'il regarde comme contraires au christianisme, Luther demande, naturellement, une étude approfondie de la Bible. De plus, la doctrine du sacerdoce universel induit, elle aussi, un accès direct à la Bible pour tous les fidèles. D'ailleurs, dans son célèbre appel Aux magistrats de toutes les villes allemandes pour les inviter à ouvrir et à entretenir des écoles chrétiennes, Luther insiste beaucoup, dès 1524, sur l'importance de l'instruction et sur la nécessité d'une forte culture classique, non pas dans un but simplement scientifique, mais comme instrument au service de l'Église et de l'État, puisque, selon lui, un État sera beaucoup mieux gouverné par des hommes instruits que par «des butors et des rustres»(2). Il souligne l'importance d'une bonne connaissance des langues anciennes, l'hébreu, le grec et le latin, seule façon selon lui de parvenir à une véritable connaissance de l'Évangile:

(1) François Furet, Jacques Ozouf, Lire et écrire, l'alphabétisation des Français de Calvin à Jules Ferry, Paris, les Éditions de minuit, 1977, vol. 1, p. 71.

(2) Martin Luther, Euvres (publiées sous les auspices de l'Alliance nationale des Églises luthériennes de France et de la revue Positions luthériennes), Genève, Labor et Fides, 1958, vol. 4, p. 103. Il ajoute, avec son style bien connu: "Qu'on prenne donc plutôt pour maitres des truies et des loups et qu'on les établisse sur ceux qui ne font pas attention à la façon dont ils sont gouvernés par des hommes» (ibid.). 
«[...] bien que l'Évangile soit venu et vienne chaque jour par le Saint-Esprit, c'est néanmoins par le moyen des langues qu'il s'est répandu, et c'est aussi par ce même moyen qu'il doit être conservé. [...] Qu'on le sache bien, sans les langues nous ne conserverons certainement pas l'Évangile. Les langues sont les fourreaux dans lesquelles est logée cette lame de l'Esprit. Elles sont le coffre dans lequel on porte ce trésor. Elles sont le vaisseau dans lequel on enferme ce breuvage. Elles sont les celliers où l'on resserre cette nourriture $\gg(1)$.

Mais Luther ne se contente pas d'insister sur la nécessaire création d'écoles classiques destinées à la formation des intellectuels et des élites sociales, il va beaucoup plus loin en réclamant la mise sur pied d'écoles pour les enfants du peuple, parce que «c'est le commandement de Dieu ». Certes, Dieu demande que les parents instruisent leurs enfants, mais Luther fait remarquer que la plupart des parents ne sont pas en mesure de le faire: ils ne sont ni assez conscients, ni assez qualifiés et ils manquent de temps. De plus, Luther ne fait pas de distinction entre les garçons et les filles et souhaite des écoles pour tous les enfants (2). Présentant tout un programme de développement de l'instruction, Luther induit donc un rapport particulier au livre lui-même en précisant:

«[...] que tous ceux qui ont à cœur l'amour et le désir de voir instituer et maintenir ces écoles et cette étude des langues en Allemagne, réfléchissent à la nécessité de n'épargner ni le zèle, ni les frais, pour créer de bonnes bibliothèques ou maisons des livres, principalement dans les grandes villes qui sont susceptibles de le faire. Car si l'on veut que l'Évangile et tous les arts subsistent, il faut qu'ils soient rédigés par écrit dans des livres et rassemblés, ainsi que l'ont fait les prophètes et les apôtres [...]. Et cela non seulement pour que ceux qui doivent nous diriger spirituellement et temporellement aient de quoi

(1) Ibid., p. 105.

(2) Sachant que les enfants doivent «vaquer au travail à la maison», il précise: «Mon idée la voici: qu'on envoie les garçons chaque jour une heure ou deux, dans telle école et qu'on les fasse néanmoins travailler le reste du temps à la maison, apprendre un métier ou ce à quoi on les destine [...]. De même, une petite fille dispose d'assez de temps pour aller chaque jour une heure à l'école et s'acquitter quand même de sa tâche à la maison. [...] La seule chose qui manque, c'est le sérieux désir d'éduquer la jeunesse et d'aider et assister le monde en lui donnant des gens comme il faut. Le diable préfère de beaucoup les grossiers lourdauds et les gens inutiles afin que les hommes ne soient pas trop heureux sur terre» (ibid., pp. 112-113). 
lire et étudier, mais aussi pour que l'on conserve les bons livres et qu'ils ne se perdent pas avec les langues et les arts que nous avons maintenant reçus de la grâce de Dieu. Ici aussi, saint Paul a fait preuve de zèle, lorsqu'il recommande à Timothée de s'appliquer à la lecture et lorsqu'il lui recommande aussi d'apporter le parchemin laissé à Troas»(1). Et Luther d'expliciter le type de livres qui devraient se trouver dans les bibliothèques.

On le voit, c'est bien la conjonction du sola scriptura et du sacerdoce universel - l'un et l'autre principes constitutifs de la Réforme qui conduit Luther à réclamer le développement de l'instruction de tout le peuple chrétien. Ce qui est logique: Luther fait appel à l'Écriture contre le magistère de l'Église romaine; dans ce système de pensée, il est indispensable que le peuple connaisse la Bible, devenue la seule autorité (2). D'ailleurs l'appel de Luther est entendu par une bonne partie des princes et des «magistrats» des villes libres allemandes passées à la Réforme.

Après Luther, nombre d'autres Réformateurs, Zwingli et Bucer notamment, tentent eux aussi de développer l'instruction. Quant à Calvin, qui influence beaucoup les Églises réformées de France, il cherche lui aussi à élever le niveau culturel des Genevois. En 1541, il fait adopter par les Conseils de la ville des Ordonnances ecclésiastiques qui prévoient, entre autres, qu'il y aura des bacheliers affectés à l'instruction des enfants, tout en étant soumis à la discipline ecclésiastique au même titre que les pasteurs. Ce qui montre l'importance qu'il attache à enseignement.

(1) Ibid., pp. 114-115.

(2) Luther affirme: «Le pouvoir du pape est analogue à tout autre pouvoir. Respectable donc comme tout pouvoir tant qu'il reste ordonné à sa fin qui est le bien de l'Église et la prédication de la parole de Dieu. Ce n'est pas Pierre [c'est-à-dire l'appartenance à une institution se présentant comme fondée par l'apôtre Pierre], c'est la foi qui fonde l'Église»; cité par Pierre Chaunu, Le temps des réformes, Paris, Fayard, 1975, pp. 447-448. Comme l'écrit le cardinal Ratzinger, devenu depuis le pape Benoît XVI: «Luther a supprimé la frontière existant entre enseignement de l'Église et recherche théologique. Un enseignement de l'Église qui est contredit par des évidences exégétiques n'est pas, selon lui, enseignement d'Église. [...] L'évidence de l'exégèse remplace chez lui le pouvoir du magistère, le magistère est celui du docteur et de personne d'autre»; cité par Jean-Paul Willaime, La précarité protestante, Genève, Labor et Fides, 1992, p. 21. 


\section{Réforme, livres et lecteurs}

En fait, le lien entre la Réforme et l'éducation est double, parce que les Réformateurs ne réclament pas seulement le développement de la culture profane. Le type d'instruction religieuse dont ils se font les champions est lui aussi porteur d'une alphabétisation du peuple: dès lors que la Bible est la seule autorité (et non plus la hiérarchie de l'Église) et que la lecture individuelle de la Bible est une exigence fondamentale, il est indispensable que chaque fidèle soit en mesure de la lire et donc qu'il dispose d'un minimum d'instruction. D'ailleurs, au milieu du $\mathrm{XVI}^{\mathrm{e}}$ siècle, les régents d'école sont nombreux à adhérer à la Réforme, et ils sont parmi ses principaux propagateurs. Et, pour développer l'influence du protestantisme, l'un des moyens souvent utilisés alors - lorsque les huguenots dominent socialement une région - est l'introduction de maîtres protestants dans les écoles. De plus, chacun sait que la Discipline des Églises réformées de France du XVI $I^{\mathrm{e}}$ siècle fait l'obligation aux Églises de «dresser» une école à côté de chaque Église locale, le maitre devenant l'auxiliaire du pasteur. Et, comme le précise Marianne Carbonnier-Burkard, cette même Discipline demande aux fidèles d'avoir «au moins un psautier par famille, contenant les 150 psaumes traduits et versifiés par Marot et Bèze, avec à la suite le Catéchisme de Genève, quelques prières, la liturgie de Genève et la Confession de foi de La Rochelle» (1).

Naturellement, tous les paysans pauvres des montagnes des Cévennes ou du Vivarais, ainsi que ceux des régions marécageuses de la presqu'île d'Arvert, par exemple, n'ont pas les moyens de s'acheter des livres. Mais lorsque cela leur est matériellement possible, bien des protestants achètent des livres et les transmettent à leurs enfants. Ainsi, dans son étude sur les bibliothèques de Metz au XVII ${ }^{\mathrm{e}}$ siècle, Philip Benedict (2) montre que $80 \%$ des familles protestantes ont des livres, alors que ce n'est le cas que de $20 \%$ des familles catholiques, et que les familles protestantes possèdent nettement plus de livres que les familles catholiques (3). Naturellement, parmi les livres les plus

(1) Marianne Carbonnier-Burkard, Patrick Cabanel, Une histoire des protestants en France, Paris, Desclée de Brouwer, 1998, p. 72.

(2) Philip Benedict, «Bibliothèques protestantes et catholiques à Metz au XVII ${ }^{e}$ siècle», Annales ESC, mars-avril 1985, pp. 343-370.

(3) Chez les artisans et les marchands qui ont des livres, les inventaires après décès montrent que les protestants en possèdent 6 en moyenne et les catholiques 2,5; chez les avocats, magistrats et médecins, les chiffres sont respectivement de 124 et de 37. 
souvent présents dans les successions des protestants, on trouve la Bible de Genève ou le Nouveau Testament; ces volumes sont transmis de père en fils, comme le montre la page de garde où sont inscrites les naissances et les morts des générations successives.

Il est clair qu'une culture biblique fondée sur une lecture fréquente de la Bible, familiale ou individuelle, est longtemps un des signes distinctifs de l'appartenance au protestantisme. Un exemple parmi bien d'autres: en tournée de prédication dans le Vivarais en 1841, un pasteur suisse nommé François Delétra note dans son Journal, à propos des protestants du Chambon-sur-Lignon:

«Tous les gens de la montagne [de la Haute-Loire] connaissent la Bible ou au moins le Nouveau Testament mieux que moi [il est pasteur!] car il n'y a que ce livre dans les écoles. Ils se disputent souvent dans les cabarets avec des volées de passages. [...] Tout le monde lit sa Bible, tout le monde bénit sa table, tout le monde vient au temple» (1).

Il ne faut certes pas généraliser et Delétra signale aussi des régions du Vivarais où la Bible est peu lue par les protestants. Il reste que ce témoignage est corroboré par d'autres, en particulier celui de François Bureau, né en 1837 et fils d'un pauvre journalier d'une petite commune au sud des Deux-Sèvres (Celles-sur-Belle), devenu instituteur, puis pasteur, à force d'acharnement au travail et d'attachement à la culture, le tout soutenu par une foi chevillée au corps. Il écrit, à propos des paysans poitevins du milieu du XIX siècle, qui vivent dans une région rurale un peu à l'écart des grands courants commerciaux et intellectuels et sont en général peu fortunés, mais qui rejoignent dans leur rapport aux livres les pratiques des marchands et des artisans protestants de Metz au XVII ${ }^{e}$ siècle étudiés par Philip Benedict:

«Un certain Massé de Villermach [Villermat, commune de Beaussais, non loin de Celles-sur-Belle] savait par cœur toute la Sainte Écriture; aussi était-il surnommé la Bible; d'autres la lisaient souvent. Les Bibliothèques de famille se composaient outre le livre saint, d'un psautier, d'un recueil de prières pour toutes les circonstances de la vie et d'un sermonnaire. Ces livres venaient des pères.

(1) François Delétra, «Journal de ma tournée de prédication dans le Vivarais en 1841 », Bulletin de la Société de l'histoire du protestantisme français, juillet-septembre 1982, pp. 400-402. 
Ils étaient placés sur un meuble appelé le cuilleré, suspendu au plafond (1). Ces bons ouvrages étaient surtout lus le dimanche, dans les veillées d'hiver et dans les jours de deuil. Les anciens de l'Église [on dit aujourd'hui les conseillers presbytéraux] avaient encore une liturgie qui leur permettait de faire les enterrements à la place du pasteur» (2).

Jean Rivierre, qui édite ce texte, précise qu'en 1935 il a organisé une exposition de livres anciens à Chey (non loin de Celles-sur-Belle) et qu'à son grand étonnement, il en a trouvé «dans presque toutes les maisons; en très peu de temps avaient été rassemblés trente éditions de la Bible, quarante psautiers différents et des dizaines de sermonnaires, en tout plus de 250 titres» (3).

En fait, il est clair que l'une des réussites de la Réforme a été de créer un type de piété marqué par un rapport au livre bien différent de celui des catholiques. Ce genre de rapport à l'écrit caractérise l'ensemble des protestants, mais, en France, il est renforcé par les vicissitudes de l'histoire. Chacun le sait, au moment de la révocation de l'Édit de Nantes, Louis XIV tente d'éradiquer le protestantisme en utilisant tous les moyens, même les plus violents, mais aussi en faisant disparaître le corps pastoral (surtout par exil). Raisonnant en catholique, il pense qu'en privant les protestants de leurs pasteurs, il parviendra à faire disparaître les Églises réformées. Mais il oublie alors que les protestants, qui adhèrent à la doctrine du sacerdoce universel, peuvent se passer de pasteur, puisque la famille protestante est une «petite Église». L'absence de pasteur conduit donc, le plus souvent, les protestants français (en dehors d'assemblées «du Désert», épisodiques) à se replier sur une pratique religieuse familiale, centrée sur le culte de famille, lui-même, pour l'essentiel, organisé autour de la lecture de la Bible familiale. Dans Si le grain ne meurt, André Gide en a laissé une description intéressante, datée de la fin du XIX ${ }^{\mathrm{e}}$ siècle, alors que l'exercice du culte est bien évidemment devenu libre; mais cela n'a pas fait disparaître les habitudes de pratique religieuse fami-

(1) À l'origine le «cuilleré» est une planche de bois percée de trous où chaque membre de la famille place sa cuiller après le repas, et qui est suspendue au plafond par des cordes placées au-dessus de la table. On y dépose aussi les livres possédés par la famille parce que, sur cette planche suspendue, ils sont inaccessibles aux rongeurs friands de papier, qui sont alors assez nombreux dans les fermes.

(2) «Mémoires du pasteur François Bureau (1837-1905)», Bulletin de la Société de l'histoire du protestantisme français, octobre-décembre 1966, p. 412.

(3) Ibid., note 78. 
liale. Égaré à la tombée de la nuit dans la campagne près d'Uzès, il frappe à la porte d'un mas, pour ne pas passer la nuit à la belle étoile. Il est accueilli et, remarquant «sur une sorte d'étagère une grosse Bible», il comprend qu'il s'agit d'une famille protestante, puisque c'est alors un marqueur religieux évident. Il dîne avec la famille puis il participe au culte de famille:

«Mais, dit le vieux [le grand-père, puisque le père est absent], vous permettrez que nous ne dérangions pas nos habitudes [...] Alors il alla chercher la grosse Bible que j'avais entrevue, et la posa sur la table desservie. Sa fille et ses petits-enfants se rassirent à ses côtés devant la table dans une attitude recueillie qui leur était naturelle. L'aïeul ouvrit le livre saint et lut avec solennité un chapitre des évangiles, puis un psaume; après quoi chacun se mit à genoux devant sa chaise, lui seul excepté que je vis demeurer debout, les yeux clos, les mains posées à plat sur le livre refermé. Il prononça une courte prière d'action de grâce, très digne, très simple, sans requêtes, où je me souviens qu'il remercia Dieu de m'avoir indiqué sa porte, et cela d'un tel ton que tout mon cœur s'associait à ses paroles. Pour achever il récita le «Notre Père», puis il y eut un instant de silence, après quoi chacun des enfants se releva» (1).

Ce texte possède certes un caractère littéraire, mais il traduit aussi une réalité: l'attachement des protestants à une piété familiale fondée sur la lecture de la Bible. Et donc un rapport particulier au livre et à la lecture.

On peut toutefois se demander si ces caractéristiques, forgées surtout $\mathrm{du} \mathrm{XVI}^{\mathrm{e}}$ au $\mathrm{XVIII}^{\mathrm{e}}$ siècle, conduisent les protestants français à conserver une attitude spécifique en face des livres et de l'instruction après la Révolution française, alors qu'ils jouissent de la liberté religieuse, que l'amélioration des techniques d'impression rend les livres moins coûteux (ce qui leur confère aussi un moindre prestige) et que l'État entreprend d'alphabétiser l'ensemble de la population. On peut sans doute, pour l'anecdote, remarquer qu'en 1833, c'est le protestant François Guizot qui est l'auteur de la première grande loi destinée à permettre un véritable développement de l'instruction primaire. Mais une étude de cette loi montre bien qu'elle n'a rien de spécifiquement «protestant» et que Guizot, champion de l'usage de la raison dans le domaine politique, sait qu'une loi «protestante» n'aurait aucune

(1) André Gide, Si le grain ne meurt, Paris, Gallimard, coll. «Folio», 1974, pp. 43-44. 
chance d'être mise en application dans un pays catholique comme la France. D'ailleurs, sous la Restauration, bien des ecclésiastiques catholiques hostiles à la méthode d'enseignement mutuel n'ont pas manqué de lui reprocher d'être une méthode «protestante», qu'il ne convenait pas d'utiliser en France.

\section{De Pestalozzi à Jules Ferry}

Dès lors, et nous croyons inutile d'y insister beaucoup, le système scolaire mis en place par l'État à partir des années 1830 s'est bâti selon deux logiques opposées, mais qui cherchaient l'une et à l'autre à consolider cette paix confessionnelle dont le destin avait été si fragile dans l'histoire de la France. Jusqu'au début des années 1880, c'est une logique de confessionnalisation de l'école publique qui l'a emporté: aux côtés d'écoles publiques catholiques, avec des maîtres, des élèves, des prières et des catéchismes catholiques, coexistaient des écoles publiques protestantes, avec des maîtres, des élèves, des catéchismes protestants. La laïcisation des programmes et des personnels, par les lois Ferry et Goblet, en 1882-1886, a changé la donne, en installant dans un même lieu et devant un même programme des enfants venus de différentes confessions, mais invités à en déposer les signes et saveurs aux patères du couloir avant d'entrer dans cet espace utopique qu'entend être la classe - la laïcité est à la fois une pratique et une utopie, dont elle emprunte la volonté d'établir des lieux radicalement neutres et vides à moins de les remplir de ses propres icônes, Marianne, système métrique, cartes géographiques barrées de noir ou de rose, etc. Cette laïcité à la Jules Ferry, désormais bien campée dans notre légendaire national, n'a pourtant échappé ni aux contestations, ni aux dérives, ni aux échecs. Une forte tentation laïciste, à partir des années 1902-1904, a conduit à la fois à évider l'école publique de toute attention au religieux - la célèbre «laïcisation» de La Fontaine ou du Tour de la France par deux enfants - et à interdire tout enseignement aux congrégations religieuses. Cette dernière mesure a entrâné l'exil de milliers de religieuses et frères enseignants, mais aussi la constitution d'un enseignement catholique non congréganiste, prédécesseur direct de celui que nous connaissons (1). Après la tourmente du début du

(1) André Lanfrey, Sécularisation, séparation et guerre scolaire. Les catholiques français et l'école (1901-1914), Paris, Cerf, 2003; Patrick Cabanel, Jean-Dominique Durand (dir.), Le grand exil des congrégations religieuses françaises 1901-1914, Paris, Cerf, 2005. 
siècle, puis la reconnaissance, encore timide, que pratique le régime de Vichy en lui accordant des subventions publiques, cet enseignement catholique a bénéficié sous les $\mathrm{IV}^{\mathrm{e}}$ et $\mathrm{V}^{\mathrm{e}}$ Républiques de lois décisives pour son financement et son institutionnalisation, même si la guerre scolaire n'a pratiquement jamais pris fin jusqu'aux années 1990. Dans ce contexte rapidement évoqué, quelle a été la place du petit protestantisme français, qui n'a jamais pesé beaucoup plus de $2 \%$ de l'ensemble de la population? C'est à cette question que les articles que nous avons réunis entendent apporter des réponses, pour une période qui va de la fin du XVIII ${ }^{\mathrm{e}}$ siècle au vote de la loi Debré en 1959.

Nous venons de rappeler combien le protestantisme est attentif, d'abord pour des raisons théologiques, à l'alphabétisation des siens. À cet égard, le pays de Montbéliard offre une vérification expérimentale particulièrement convaincante; elle vaut moins, du reste, pour la France protestante «de l'intérieur» (poitevine et méridionale) que pour l'ensemble de l'Europe luthérienne, dont Montbéliard est la pointe avancée vers le sud et l'ouest. L'article d'Élisabeth Berlioz donne à voir une situation doublement exceptionnelle dans notre pays, puisqu'il n'y est question ni de la confession dominante, ni du protestantisme dominant, mais exemplaire à l'échelle des campagnes prussiennes ou scandinaves. On voit combien les pasteurs, parfaits disciples de Luther, sont attentifs à la scolarisation des enfants, condition de leur évangélisation, et combien ils se passionnent pour les questions de pédagogie, cette science largement apparue dans l'Europe protestante germanique. Inutile de préciser que pasteurs et éducateurs du pays de Montbéliard sont bien éloignés de toute laïcité à la française, en particulier sous la Révolution: leur école ne peut être que chrétienne. Si la laïcité est à inventer dans la France du $\mathrm{XIX}^{\mathrm{e}}$ siècle, ce n'est à l'évidence pas dans cette région protestante, et pas plus dans les Cévennes, mais en région de dissémination protestante, ce qui change radicalement les données du problème. On ajoutera que, comme le remarquait Quinet pour la Suisse, en pays protestant l'instituteur n'est ni l'adversaire ni le subordonné du pasteur, les deux hommes collaborant à un même objectif, alors que la France du Second Empire a vu croître l'irritation anticléricale du monde enseignant à l'encontre d'un clergé comblé par la loi Falloux et la manière dont l'État bonapartiste appliquait cette loi. 
Attentifs aux leçons et aux recettes qu'ils pouvaient recevoir de l'Europe protestante, politiques et pédagogues de la génération de Jules Ferry ont choisi pour maître, sans cesse célébré, véritable «Réformateur» européen au tournant des $\mathrm{XVIII}^{\mathrm{e}}$ et $\mathrm{XIX}^{\mathrm{e}}$ siècles, le Suisse de langue allemande Pestalozzi. Ce faisant, il est vrai, la République ignorait en grande partie (1) une figure française cardinale quant aux liens qui se tissent entre le pasteur et l'instituteur, l'évangélisation et l'alphabétisation, celle de Jean-Frédéric Oberlin (1740-1826) dans sa communauté du Ban-de-la-Roche. Mais Oberlin n'était qu'un obscur pasteur de campagne, aujourd'hui encore inconnu d'un public qui ne serait ni protestant ni alsacien, alors que Pestalozzi avait joui d'un rayonnement européen, publiant un ouvrage, Léonard et Gertrude (2), admiré presque à l'égal de l'Émile et fournissant leurs cadres aux premières écoles normales d'instituteurs fondées par la Prusse aux lendemains de Iéna, au temps des Fichte et des Humboldt. Une Prusse dont la France républicaine allait, au lendemain de Sedan, méditer l'exemple et imiter les leçons de reconquête par l'expansion intérieure, celle des esprits à instruire. Certes, il ne s'agissait pas de s'inspirer du chrétien Pestalozzi, mais du pédagogue Pestalozzi, évidemment issu de l'Europe protestante comme allaient l'être d'autres pédagogues auxquels le Dictionnaire de pédagogie et la Revue pédagogique ont offert des tribunes, les Allemands Diesterweg, Basedow et Froebel, l'Écossais Blackie, mais aussi, par-delà les frontières du temps ou de l'océan, le hussite Comenius (Komenski) ou les Américains Cyrus Pierce et Horace Mann. Kant n'est jamais très éloigné dès lors qu'il s'agit de définir une morale laïque, un Kant dont deux des trois principaux passeurs en France, Charles de Villers au début du XIX ${ }^{\mathrm{e}}$ siècle, Charles Renouvier dans son dernier tiers, étaient des proches du protestantisme, le premier par ralliement intellectuel, le second par conversion philosophique et religieuse (3).

(1) Mais pas totalement: le Dictionnaire de pédagogie lui consacre une notice, sous la signature du pasteur Tommy Fallot (I, 2, 1887, pp. 2127-2132); mais la notice de Pestalozzi, rédigée dans le même volume par James Guillaume, couvre les pages $2283-2358 \ldots$

(2) Lienhard und Gertrud, ein Buch für das Volk, 1781, traduit deux ans plus tard en français, Léonard et Gertrude, ou les Mours villageoises, telles qu'on les retrouve à la Ville et à la Cour, Histoire morale traduite de l'allemand par Louis-Esaïe Pajon de Moncets, Berlin, G.J. Decker, 1783.

(3) Le troisième est le républicain Jules Barni, réfugié à Genève sous le Second Empire. 
Que Pestalozzi ait été un jalon inattendu, mais peut-être capital, dans la généalogie de l'école républicaine - nous ne disons pas encore «laïque» - trouve une étrange vérification biographique dans le cas d'Élie Rabier, le presque inamovible directeur de l'enseignement secondaire dans la République de Ferry, qui est l'arrière-petitfils d'un élève du pédagogue suisse venu fonder une école à Bergerac à l'invitation du philosophe Maine de Biran. Rabier est l'un des «directeurs» protestants de la laïcité en cours d'institutionnalisation, aux côtés de Ferdinand Buisson (qui dirige l'enseignement primaire), de Louis Liard (qui est à la tête de l'enseignement supérieur l'homme n'est qu'un compagnon de route du protestantisme) (1), de Félix Pécaut puis Jules Steeg à Fontenay-aux-Roses, de $\mathrm{M}^{\mathrm{me}}$ Jules Favre à Sèvres, de Pauline Kergomard (née Reclus) à la tête des jeunes écoles maternelles, etc. (2). Ces hommes et femmes, pour certains anciens pasteurs ou théologiens, pour d'autres membres de familles pastorales, incarnent ce moment unique, et peut-être provisoire et fragile, où l'histoire de France n'a plus été écrite ni par les héritiers de Bossuet, ni par ceux de Voltaire, mais bien par les arrièrepetits-fils d'un Calvin ou peut-être d'un Castellion, ce théologien protestant libéral qui fut l'adversaire malheureux du prédicateur de Genève et auquel Ferdinand Buisson trouva le temps de consacrer en 1892, à l'âge où les grands commis de l'État ont cessé de tels apprentissages, une thèse puissante et militante. Bien sûr, il était impensable de consacrer à notre tour des études à chacune de ces personnalités, en particulier au même Buisson qui a bénéficié d'un vrai mouvement collectif de redécouverte depuis une vingtaine d'années (3). À moins

(1) Au lendemain des obsèques présidées par le pasteur Roberty, en 1917, Pauline Kergomard écrit: «Je ne savais pas qu'il eût adhéré en quoi que ce soit au protestantisme; il paraît que c'est au moment où sa fille est sortie des langes qu'il l'a fait baptiser par un pasteur»; cité par Geneviève et Alain Kergomard, Pauline Kergomard, Rodez, Fil d'Ariane éditeur, 2000, p. 250. Dans une lettre félicitant Steeg pour sa nomination à l'inspection générale, en mars 1890, Élie Pécaut, un fils de Félix, écrit: «Quelle précieuse recrue vous êtes pour l'enseignement primaire, où vous confirmerez encore les efforts de tout ce petit groupe d'esprit protestant, mon père, Buisson, Liard, qui en est en somme tout inspiré»; cité par Lucien Carrive (éd.), Un pasteur républicain au XIXe siècle. Lettres de Jules Steeg à Maurice Schwalb 1851-1898, Paris, Presses de la Sorbonne nouvelle, 1993, pp. 382-383.

(2) Pour une vision d'ensemble, Patrick Cabanel, Le Dieu de la République. Aux sources protestantes de la lä̈cité (1860-1900), Rennes, Presses universitaires de Rennes, 2003.

(3) Avec pas moins de deux thèses, celles de Mireille Gueissaz-Peyre, L'image énigmatique de Ferdinand Buisson. La vocation républicaine d'un saint puritain, Villeneuve d'Ascq, Presses du Septentrion, 2002, et de Samuel Tomei, Ferdinand 
qu'il ne s'agisse «du» Buisson, ce Dictionnaire de pédagogie déjà cité et dont nous savons aujourd'hui à peu près tout, y compris grâce à la présente revue (1). Cette redécouverte par l'historiographie, également remarquable dans le cas de Renouvier (2), est sans doute le signe d'une nation soucieuse de mieux connaître la généalogie de sa laïcité. Il nous a paru suffisant, ici, de proposer un portrait de l'un de ces «directeurs» protestants de l'école laïque, et Yves Verneuil a donc retenu Élie Rabier.

À lire son article, ainsi que celui de Jacqueline Gautherin, qui ne manque du reste pas de croiser Rabier, une question vient à l'esprit: en quoi le personnage illustre-t-il, au-delà d'une origine et de tout ce qu'elle peut supposer de hasard et de répétition, les liens entre le protestantisme et l'école? Question redoutable, mais somme toute banale, que trouvent sur leur route historiens et sociologues dès lors qu'ils s'intéressent aux identités et à ce que Max Weber, le premier, appela les «affinités électives» entre une religion, ou son éthique, et tel ou tel domaine de la vie sociale. Que nous apprennent, à cet égard, la personnalité et le parcours d'Élie Rabier? Si le protestant est

Buisson (1841-1932). Protestantisme libéral, foi lä̈que et radical-socialisme, Lille, Atelier national de reproduction des thèses, 2 vol., 2005. Voir aussi Pierre Hayat, $L a$ Passion laïque de Ferdinand Buisson, Paris, Kimé, 1999, et Laurence Loeffel, Ferdinand Buisson, apôtre de l'école lä̈que, Paris, Hachette, 1999.

(1) Patrick Dubois, «Le Dictionnaire de F. Buisson et ses auteurs (1878-1887)», Histoire de l'Éducation, ${ }^{\circ}$ 85, janvier 2000, pp. 25-47. Du même, Le Dictionnaire de Ferdinand Buisson. Aux fondations de l'école républicaine (1878-1911), Berne, Peter Lang, 2002, et, en collaboration avec Annie Bruter, Le Dictionnaire de pédagogie et d'instruction primaire de Ferdinand Buisson. Répertoire biographique des auteurs, Paris, INRP, 2002. Daniel Denis, Pierre Kahn (dir.), L'école républicaine et la question des savoirs. Enquête au cœur du Dictionnaire de pédagogie de Ferdinand Buisson, Paris, CNRS Éditions, 2003. Daniel Denis, Pierre Kahn (dir.), L'École de la Troisième République en questions. Débats et controverses dans le Dictionnaire de pédagogie de Ferdinand Buisson, Berne, Peter Lang, 2006.

(2) Signalons notamment les travaux de Marie-Claude Blais, Au principe de la République. Le cas Renouvier, Paris, Gallimard, coll. «Bibliothèque des idées », 2000; Laurent Fédi, «Philosopher et républicaniser: la Critique philosophique de Renouvier et Pillon, 1872-1889», Romantisme, revue du dix-neuvième siècle, 115, premier trimestre 2002, pp. 65-82; Fernand Turlot, Le personnalisme critique de Charles Renouvier. Une philosophie française, Strasbourg, Presses universitaires de Strasbourg, 2003; le numéro 45 de Corpus, revue de philosophie: "Renouvier: philosophie politique», $4^{\mathrm{e}}$ semestre 2003; et les rééditions du Petit traité de morale à l'usage des écoles primaires laïques (INRP, 2003, présenté par M.-Cl. Blais), de la Science de la morale (1869, 2 vol.) et de l'Uchronie (1876), repris tous deux dans le Corpus des œuvres de philosophie en langue française (Fayard, respectivement en 2002 et 1988). 
d'abord celui que ses adversaires ou «les autres» désignent comme tel, à la manière du juif pour Sartre, Rabier en est bien un: on verra surgir à son encontre quelques clichés éculés, l'austérité du huguenot, la froideur du clergyman... S'il est celui dont des solidarités de minoritaires permettent d'accélérer la carrière, force est de reconnaître avec l'auteur que rien ne permet de l'avancer, ni d'ailleurs de le réfuter. Ses liens avec un Buisson, un Pécaut ou un Liard sont inévitables dès lors qu'il occupe la place qui est la sienne. Quant à imaginer un homme qui puiserait dans sa foi ou sa culture protestante des principes d'action et de réflexion, l'exercice est pour le moins acrobatique. Il n'y a aucune façon protestante, ni catholique, de diriger l'enseignement secondaire, sauf à placer systématiquement des coreligionnaires aux meilleurs postes ou à peser sur le choix des auteurs classiques - un Agrippa d'Aubigné plutôt qu'un Ronsard -, mais même un Maurras n'a pas feint de défendre une telle thèse.

Jacqueline Gautherin affronte les mêmes questions, pour ne pas dire les mêmes apories, dans son enquête sur les premiers spécialistes universitaires de la Science de l'éducation: outre que les protestantismes des intéressés sont très divers, et parfois même incompatibles (souvenons-nous de la grande querelle entre les «évangéliques» et les «libéraux»), rapporter toute une identité à la seule dimension confessionnelle conduit à oublier que bien d'autres origines, formations et réseaux concourent à faire et à dire ce qu'est un homme. Camarades de promotion à l'École normale et tous deux agrégés de philosophie, Liard et Rabier sont peut-être plus liés par ces deux titres que par un protestantisme qui est de ralliement chez l'un et de famille chez l'autre. En revanche, Jacqueline Gautherin parvient à identifier, notamment chez Ferdinand Buisson et chez Gaston Richard, «sociologue» dissident (il a rompu avec Durkheim), une possible manière protestante de concevoir la pédagogie, qu'elle qualifie de puéro-centrisme: l'éducation y est «fondée sur l'assurance de la perfectibilité humaine et centrée sur la personne de l'enfant». L'historien de la littérature trouverait d'intéressantes confirmations de cette thèse dans un livre aujourd'hui un peu injustement oublié de Paul Hazard, Les livres, les enfants et les hommes, qui ne manque pas d'opposer les conceptions «nordique» et «latine» de l'enfance (1).

(1) Paris, Flammarion, 1932. Le livre III, «De la supériorité du Nord sur le Midi », est à méditer, en dépit d'un titre et d'une approche évidemment vieillis. 


\section{Une laïcité problématique?}

Quoi qu'il en soit, l'historien se doit de dépasser le cas d'un ou de quelques individus, pour envisager toute une génération et, mieux encore, le moment dont il a été parlé plus haut et qui a fait que, pour des raisons multiples, des protestants, chrétiens sans cléricalisme, laïques sans athéisme, ou du moins tenus pour tels, ont très exactement fourni the right men in the right place. Protestantisme et laïcité, donc. Mais cette «équation» mérite d'être réinterrogée, à la lumière de trois articles dont les auteurs, sans s'être concertés, aboutissent à des constatations étonnamment voisines quoique portant sur près de cent cinquante ans d'histoire, ceux de Patrick Cabanel sur la Société pour l'encouragement de l'instruction primaire parmi les protestants de France (1) - en amont des lois Ferry -, d'Arnaud Baubérot et d'André Encrevé sur les Membres et amis chrétiens de l'enseignement et la Fédération protestante de l'enseignement - en aval des mêmes lois. Première constatation, qui va à l'encontre d'une certaine «histoire sainte» du protestantisme et de la laïcité, mais qui ne peut qu'agréer aux historiens: les protestants n'ont pas été «naturellement» laïques, pas plus en matière d'école que d'État, comme le montre le fait que plusieurs pays protestants européens ont encore aujourd'hui des Églises «établies». Ils se sont ralliés à la laïcité de l'école parce que, dans un pays où ils sont l'infime minorité, elle offrait les plus grandes garanties de protection et de liberté face à un péril clérical, ou tout simplement catholique, dont on les voit exprimer la crainte jusqu'à la fin des années 1950 ! Qu'ils aient conçu pour cette laïcité une reconnaissance fondamentale, pour ne pas dire viscérale, et qu'ils l'aient servie avec passion, hussards parmi les hussards; qu'ils aient apprécié de trouver entre la structure de leurs Églises et celle de la République une règle de fonctionnement commune, cellelà même de la démocratie représentative, et qu'ils l'aient fait savoir, n'enlève rien au fait que cette rencontre d'une minorité et d'une idée a été un moment d'histoire, non une donnée intemporelle qui voudrait qu'il y ait des religions «laïques» et d'autres «cléricales».

On le vérifiera, notamment, en observant les réactions et les stratégies de la «centrale» scolaire protestante du XIX ${ }^{\mathrm{e}}$ siècle, la Société

(1) L'auteur n'a pu lire l'important mémoire de maîtrise de Jean-Claude Vinard, Les écoles primaires protestantes de 1815 à 1885, Institut protestant de théologie de Montpellier, 2000, 2 vol., compte rendu par Gabrielle Cadier-Rey dans le Bulletin de la Société de l'histoire du protestantisme français, 2002, 3, pp. 613-615. 
pour l'encouragement de l'instruction primaire parmi les protestants de France (SEIPP), qui n'a pas accepté sans craintes, méfiances ou arrière-pensées la vague laïcisatrice du début des années 1880 . Ce sera peut-être même une surprise, pour certains lecteurs, que de découvrir un protestantisme aussi peu spontanément favorable à la laïcité. Mais il faut ajouter que ce protestantisme a appris à redouter bien davantage, des années 1830 aux années 1870, les pressions du cléricalisme et l'intolérance tranquille d'une société restée encore profondément catholique, ce qui ne peut que faciliter son ralliement précoce et sincère au nouveau cours; et que les dirigeants de la SEIPP ne représentent plus, dans les années 1880, que l'une des sensibilités protestantes, la grande majorité des familles et même un certain nombre de pasteurs passant d'enthousiasme à l'école laïque. Ce clivage entre des dirigeants plus «religieux» et une masse franchement «laïque» se retrouve tout au long du $\mathrm{XX}^{\mathrm{e}}$ siècle. Il est tel que les responsables de la Fédération protestante de l'enseignement et le directeur gaulliste de l'hebdomadaire Réforme, le pasteur Albert Finet, sont finalement contraints, face à l'émotion suscitée par le projet de la loi Debré, en 1959, de se rallier à leur base, dont la sensibilité laïque est vive, et d'apporter leur soutien au Comité national d'action laïque. C'est que le protestantisme français n'est pas aussi uni qu'on pourrait le croire et que des clivages d'ordre ecclésiologique et sociologique, au demeurant très classiques, le traversent: les fidèles n'épousent nécessairement ni les attitudes des pasteurs, ni celles de dirigeants souvent issus de la bourgeoisie (la célèbre «Haute société protestante»). En clair, l'attachement indéfectible à l'école laïque et le vote résolument à gauche, jusqu'au Parti communiste, bien implanté dans les Cévennes au milieu du $\mathrm{XX}^{\mathrm{e}}$ siècle, sont des constantes du peuple protestant du Midi, que les pasteurs et la bourgeoisie parisienne n'ont jamais pleinement représenté (1).

Les dirigeants du protestantisme n'en ont pas moins été pleinement laïques: mais leur laïcité n'était pas celle du CNAL ou, plus en arrière, d'un Émile Combes ou d'un Édouard Herriot. L'un des auteurs du présent numéro l'a qualifiée ailleurs de «laïcité protestante» (2), sur un modèle à l'américaine: l'État et l'école sont

(1) Dès les années 1830 et 1840 , ce peuple protestant du Midi passe à la République alors que la bourgeoisie orléaniste attend les années 1870 pour se rallier.

(2) André Encrevé, «Les Protestants réformés face à la laïcisation de l'école au début des années 1880», Revue d'histoire de l'Église de France, t. 84, 1998, pp. 71-96. 
parfaitement séparés de quelque Église que ce soit, mais le christianisme protestant, ou si l'on préfère une religiosité évangélique, imprègne l'ensemble de la société. On voit bien que, des années 1870 aux années 1930 et encore dans l'après-Seconde Guerre mondiale, des protestants rêvent de faire vivre une telle laïcité, à égale distance des deux excès qui renvoient aux «deux France», le cléricalisme d'un côté, le laïcisme de l'autre. Mais l'histoire française du centre, politique ou religieux, montre combien est appelée à être laminée, en France, cette quête d'une laïcité ouverte au religieux : la puissance du refus catholique la compromet irrémédiablement, et le balancier, lorsqu'il se meut, bascule d'un inverse à l'autre, combisme au début du siècle, gaullisme catholique à partir de 1959.

Dès lors, on peut parler d'une forme d'échec du protestantisme dans sa volonté de peser en faveur d'une laïcité spiritualiste, voire d'une neutralité sincère; mais on peut tout aussi bien signaler combien les protestants se sont sentis à leur aise dans la nouvelle école laïque, à laquelle ils ont donné leurs enfants mais aussi beaucoup de maîtres, des «hussards noirs» aux inspecteurs généraux, des auteurs de manuels aux ministres (de Guizot à Jospin, en passant par Jean Zay). Ici se sont retrouvés les deux traits majeurs de leur personnalité historique: une appétence d'origine religieuse pour le livre et l'alphabétisation, mais désormais sécularisée (de la majuscule à la minuscule pour noter le «Livre»); et la chance saisie face à l'ouverture d'un espace scolaire qui ne prétend reconnaître que des individus libres et égaux, offrant par là un nouveau destin aux héritiers de minorités hier persécutées, interdites ou reléguées. La laïcité, c'est la perte du handicap pour les protestants et les juifs, et c'est la survalorisation de leur savoir-lire séculaire. Péguy, presque jaloux (mais son génie laïque et dreyfusard l'a gardé de tout dérapage), Péguy l'avait marqué, en martelant que son ami Julien Benda, le juif, lisait depuis deux mille ans et son ami Pierre-Félix Pécaut, le protestant (un fils de Félix Pécaut), depuis quatre siècles (1). Il leur était aisé de devenir les «meilleurs élèves» de la nouvelle société, pour reprendre une formule de Dominique Schnapper à propos des juifs (2), dès lors que la

(1) Charles Péguy, «Note conjointe sur M. Descartes», Euvres en prose complètes, Paris, Gallimard, Bibliothèque de la Pléiade, 1993, pp. 1296-1297.

(2) Dominique Schnapper, «Juifs hongrois et juifs français. Les destins de 1'“assimilation"», Commentaire, 87, automne 1999, p. 671 [669-675]. 
classe choisissait de ne reconnaître que les talents et le travail. Heureux comme Dieu en France, telle pourrait être la devise des juifs et des protestants dans le siècle laïque; mais c'est là un bonheur, on le vérifiera dans ce numéro, qui a rarement été sans nuages, parce que le catholicisme comme la laïcité à la française ont été tentés l'un et l'autre, à plusieurs reprises, de retourner ou d'aller sur des rivages que la minorité protestante, pour ne parler que d'elle, ne jugeait guère hospitaliers.

Patrick CABANEL

Université de Toulouse Le Mirail

André ENCREVÉ

Université de Paris XII 